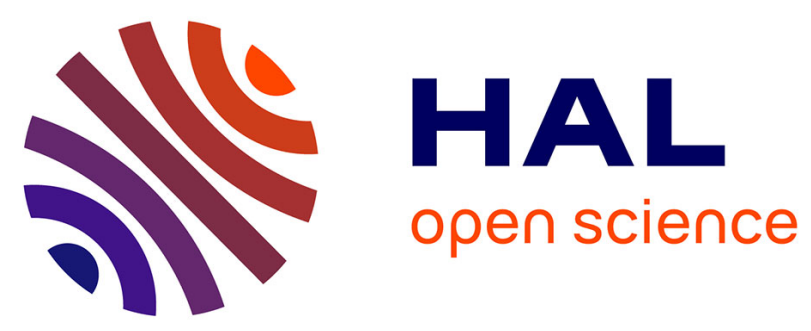

\title{
CR Julie Verlaine, Les galeries d'art contemporain à Paris de la Libération à la fin des années 1960. Une histoire culturelle du marché de l'art, 1944-1970
} Anne Gagnebien

\section{- To cite this version:}

Anne Gagnebien. CR Julie Verlaine, Les galeries d'art contemporain à Paris de la Libération à la fin des années 1960. Une histoire culturelle du marché de l'art, 1944-1970. Lectures, 2013, 10.4000/lectures.11665 . hal-03079724

\section{HAL Id: hal-03079724 https://hal.science/hal-03079724}

Submitted on 2 Feb 2021

HAL is a multi-disciplinary open access archive for the deposit and dissemination of scientific research documents, whether they are published or not. The documents may come from teaching and research institutions in France or abroad, or from public or private research centers.
L'archive ouverte pluridisciplinaire HAL, est destinée au dépôt et à la diffusion de documents scientifiques de niveau recherche, publiés ou non, émanant des établissements d'enseignement et de recherche français ou étrangers, des laboratoires publics ou privés. 


\section{OpenEdition Journals}

\section{Lectures}

Reviews

/

2013

\section{Julie Verlaine, Les galeries d'art contemporain à Paris de la Libération à la fin des années 1960. Une histoire culturelle du marché de l'art, 1944-1970}

\section{AnNe GaGnebien}

https://doi.org/10.4000/lectures.11665

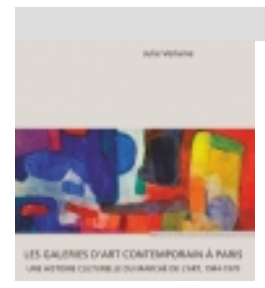

Julie Verlaine, Les galeries d'art contemporain à Paris de la Libération à la fin des années 1960. Une histoire culturelle du marché de l'art, 1944-1970, Editions de la Sorbonne, 2013, 350 p., ISBN : 978-2-85944-723-6.

You can order this publication on our partner's website Decitre

\section{Full text}

1 Peu d'ouvrages ont pris pour objet les galeries d'art françaises et se sont recentrés sur le marché plus spécifique de l'art contemporain et de ses acteurs multiples. Issu d'une thèse de doctorat soutenue en 2008, Les galeries d'art contemporain à Paris de la Libération à la fin des années 1960 retrace l'histoire sociale et culturelle du commerce de l'art de la ville de Paris depuis sa véritable émergence après la Libération à son " effondrement ", pour reprendre les mots de l'auteure, au profit d'une autre, New York dans les années soixantedix. 
2 Les marchands d'art sont ici étudiés du point de vue des relations qu'ils entretiennent avec les différents acteurs du marché, à travers le concept de " monde de l'art », les galeries étant avant tout définies comme des « espaces de sociabilité » (p. 9) où sont mis en place des " mécanismes de reconnaissance ». Ces derniers sont développés à la fois par la diffusion commerciale, l'extension internationale et l'obtention de " gratifications symboliques » des artistes. D’autres mécanismes sont également analysés comme le rôle social attribué aux galeries pour les créateurs, les experts et les publics amateurs. Loin de l'opacité, du soupçon de manipulation que ces marchands ont souhaité garder étiqueté sur leur milieu pour agir le plus librement possible, l'étude réalisée par Julie Verlaine permet de traiter des galeries d'art en tant qu'objet pour l'historien, en centrant le propos sur les femmes et les hommes amateurs et vendeurs d'œuvres d'art. Nous y retrouvons les figures emblématiques des marchands d'arts, non dénommé galeristes à l'époque tels que (pour n'en citer que quelques-un(e)s) : Denise René, Bernard Gheerbrandt, Aimé Maeght, Colette Allendy, Iris Clert, Paul Facchetti, Myriam Prévost jusqu'à Yvon Lambert. La construction et l'analyse sociologique atteste à la fois que les « marchand(e)s de tableaux » sont «plus collectionneurs que marchands » (p. 205). Les représentations et pratiques du métier où se côtoient l'argent, l'art, l'« effet d'éponymie-toponymie ${ }^{1}$ » des noms de galeries permettent d'illustrer tout autant le rôle des femmes que des hommes qui y travaillent avec passion.

3 Organisée en trois parties chronologiques, ouverte par une introduction très synthétique, cette histoire culturelle et sociale est traitée sur vingt-cinq ans à Paris (avec de nombreuses études comparées du " premier marché ${ }^{2}$ » des œuvres contemporaines en galeries, dans d'autres capitales influentes à l'époque). Fruit d'un dépouillement systématique du périodique Arts entre 1945 et 1969, croisé avec l'étude (après estimation quantitative des annuaires professionnels) des catalogues, revues, archives (souvent inédites comme des livres de comptes) de cinquante galeries 3 , ce livre, composé de trois parties, permet au lecteur de suivre les transformations du marché de l'art contemporain, d'étudier le rôle influent des galeries puis de leur évolution dans la consécration mondiale des avantgardes. La dernière partie du livre permet notamment au lecteur de suivre les nombreuses crises subies par les marchands d'art dont celle du marché de l'art entre 1962 et 1965, puis celle notable connue après 1968 quand les artistes ont voulu se " passer des galeries » (p. 17). Loin de faire une généalogie des galeries privées, cette recherche en histoire sociale est très orientée par la sociologie et l'économie de la culture (notamment la première partie sur « montée en puissance des galeries »).

4 Qualifiées ingénieusement de « club informels » (p. 10) où les membres se retrouvent par affinités sociales et esthétiques, les galeries d'art participent à divers processus explorés dans les différents axes de l'ouvrage. Deux processus qualifiant les qualités des relations entre les acteurs du milieu de l'art contemporain parisien sont relevés : celui de l'attachement et celui de la valorisation.

5 Le processus d'attachement permet d'analyser très justement ces affinités qui lient les artistes aux marchands. Il est alors observé que le marchand d'art ne choisit pas ses artistes, pas plus que ceux-ci ne choisissent leur galerie sur des critères évidents ou objectifs. L'ouvrage permet ainsi que mettre en évidence la complexité des relations entre artistes et marchands tels que le fait d'avoir des caractères compatibles, de croire en une collaboration efficace, de vouloir s'engager dans une communauté (associant amis, artistes et critiques). Le processus de valorisation se traduit quant à lui par le fait de donner un espace de visibilité publique des œuvres où elles trouvent une valeur (prix, création) et parfois même un titre, les galeries contribuent ainsi entre autres à l'authentification sociale de l'artiste.

6 Les galeries selon les analyses de l'auteure sont ainsi devenues des « institutions de fait » (p. 538) où l'intervention des marchands d'art est décisive pour les artistes (promotion, carrière des artistes et lien avec les critiques, des publics ciblés et collectionneurs, séries d'alliances qualifiées d'objectives entre elles et de consensus concentriques autour de la " Nouvelle Ecole de Paris » étiquetant de façon prestigieuse et réussie les artistes 
prometteurs de l'époque (à partir du moment où ceux-ci sont venus à Paris pour créer). Les galeries parisiennes défendent peu certains artistes comme ceux du Pop Art, ce qui traduit de grands décalages avec d'autres grandes capitales de l'art et d'une certaine manière un rétrécissement de leur influence internationale couplé de la critique d'un élitisme observé en 1968 vis-à-vis de ce microcosme spécifique. Dans un même sens, le travail d'analyse est particulièrement précis concernant la formation de la valeur artistique et des goûts bien spécifiques à cet art dit contemporain " par définition illimité et indéfini [...où] l'appréciation de ces œuvres peut encore moins se fonder sur des critères objectifs de valeurs que les jugements portant sur les œuvres plus anciennes » (p. 12).

7 Sans reprendre le travail de Howard Becker sur Les Mondes de L'art (1988), la seconde partie de l'ouvrage est originale, car elle permet de cerner le système des galeries en ayant recours au parcours biographique croisé avec la création artistique et les représentations émergentes associées de l'époque. La compréhension des stratégies économiques est pertinente pour analyser les intrications complexes entre le rôle conséquent des corporations, les stratégies professionnelles de lobbying, les réformes juridiques, taxes et licences etc. et pour comprendre les conditions plutôt favorables à leur égard - la fameuse exception culturelle française - à regarder par exemple au prisme des industries culturelles voire créatives ${ }^{4}$ dont les sciences de l'information et de la communication traitent aujourd'hui pour qualifier industrialisation de l'art et les pratiques créatives et commerciales des acteurs tout aussi présents que ne l'étaient les galeristes lors des Trente Glorieuses en France. Cependant, nous relevons que la dimension économique des œuvres est mise de côté. Elle aurait pourtant permis de saisir les efforts de popularisation, de comprendre comment se crée la cote des artistes et l'évolution de cette dernière et d'analyser les évolutions durant cette période (1944-1970). L'auteure, à juste titre, déplore de son côté le manque d'études faites entre les galeries et l'élaboration des théories de l'art, le monde de l'édition et des conservateurs qui travaillaient de pair durant la période analysée.

8 La lecture de ce livre est particulièrement jubilatoire : non seulement l'étude historique qui est faite du « système des galeries » et de leurs « passeurs » sur un quart de siècle est exceptionnelle mais les graphiques et illustrations (photographies d'époques des expositions et des galeristes et leurs amis, cartons d'invitations et archives personnelles de l'auteure) viennent ponctuer à merveille l'analyse avec des références théoriques rares et précises.

\section{Notes}

1 Le nom du marchand doit faire la réputation de la galerie, comme beaucoup qui ont subsisté aujourd'hui ont gardé celui du premier propriétaire. Est ensuite utilisé le nom du lieu de la galerie dans Paris ou le vocabulaire artistique.

2 Le " premier marché » signifie que les galeries étudiées par Julie Verlaine ne proposaient que des œuvres n' " ayant jamais fait l'objet d'une transaction commerciale » comme elle le justifie dans son introduction sur la méthode d'investigation (p. 15)

3 Julie Verlaine dénombre 168 galeries d'art moderne et contemporain en 1951 et 296 en 1971

4 Consulter à ce titre Sylvia Girel, "Philippe Poirrier (dir.), Pour une histoire des politiques culturelles dans le monde (1945-2011) », Lectures [En ligne], Les comptes rendus, 2011, mis en ligne le 20 décembre 2011, consulté le 12 mai 2013. URL : http://lectures.revues.org/7057

\section{References}

\section{Electronic reference}

Anne Gagnebien, « Julie Verlaine, Les galeries d'art contemporain à Paris de la Libération à la fin

des années 1960. Une histoire culturelle du marché de l'art, 1944-1970 », Lectures [Online],

Reviews, Online since 04 June 2013, connection on 27 January 2021. URL:

http://journals.openedition.org/lectures/11665 ; DOI : https://doi.org/10.4000/lectures.11665 


\section{About the author}

Anne Gagnebien

Docteure en sciences de l'information et de la communication, laboratoire LabSic, Paris 13

By this author

Alexis Ferrand, La formation des groupes de jeunes dans l'espace urbain [Full text]

Dominique Boullier, La ville-événement. Foules et publics urbains [Full text]

\section{Copyright}

(C) Lectures - Toute reproduction interdite sans autorisation explicite de la rédaction / Any replication is submitted to the authorization of the editors 